\title{
Programa de seguimiento de niños prematuros desde el punto de vista terapéutico y educativo
}

\author{
T. Rico Vales ${ }^{a}$, C. Herencia Solano ${ }^{b}$, A. García Martínc, \\ S. González Castroc, PJ. Puyol Build, J. Torres Mohedas ${ }^{\mathrm{e}}$ \\ assicóloga. Centro de Atención Temprana AFANDEM. Móstoles, Madrid. España. \\ ${ }^{b}$ Orientadora. Equipo de Orientación Educativa y Psicopedagógica de Móstoles, Madrid. España. \\ 'Orientadora. Centro de Atención Temprana AFANDEM. Móstoles, Madrid. España. \\ dPediatra. Servicio de Pediatría, Hospital Universitario de Móstoles. Móstoles, Madrid. España. \\ eNeuropediatra. Servicio de Pediatría, Hospital Universitario de Móstoles. Móstoles, Madrid. España.
}

\begin{abstract}
Resumen
Las necesidades de los niños prematuros son atendidas, además de por sus familias, desde diferentes servicios. Si queremos que esta atención sea adecuada, dichos servicios deben coordinarse y llevar conjuntamente programas preventivos. El programa de seguimiento de los niños nacidos prematuros en Móstoles (Madrid) se enmarca en esta coordinación imprescindible.

Pretendemos mostrar cómo se desarrolla la coordinación y qué aporta cada unidad o servicio, así como transmitir la importancia que tiene el seguimiento conjunto partiendo de una colaboración que empezó hace una década; aportamos los datos más relevantes de los resultados obtenidos.
\end{abstract}

Palabras clave: Desarrollo. Atención multidisciplinar. Prematuridad. Niños.

A preterm infants' follow up program from the therapeutical and educative point of view

Abstract

Families of preterm infants need the collaboration of different services to take care of these children. These services must coordinate to give the adequate care, implementing together preventive programs. The preterm infants' follow up program of Móstoles (Madrid) takes into account this essential coordination.

We explain how this coordination develops, what it adds to each service and the importance the joint follow up has, starting from a collaboration that has started a decade ago. We show the most relevant data of the obtained results.

Key words: Development. Multidisciplinary care. Preterm. Child.

Antonio García Martín, antvallecas@yahoo.es

Algunos datos de este artículo han sido presentados en reuniones y actividades formativas locales.

Los autores declaran no presentar conflictos de intereses en relación con la preparación y publicación de este artículo. 


\section{Introducción}

El Plan de Acción para Personas con Discapacidad (2005-2008) de la Comunidad de Madrid' tiene como objetivo "detectar precozmente a los niños con discapacidad o con problemas del desarrollo y establecer las medidas adecuadas para su seguimiento y tratamiento". Recomienda el "seguimiento durante los primeros años de vida con especial incidencia en los grupos de riesgo" para "garantizar un programa de atención integral a todos los niños con discapacidades, con alteraciones del desarrollo y riesgo de padecerlo".

Durante estos años, hemos pasado de la expresión estimulación precoz al concepto de atención temprana, es decir, hemos evolucionado desde un modelo centrado en los problemas del niño a un paradigma biopsicosocial centrado en el niño, la familia y el entorno. Esta perspectiva global implica un trabajo multidisciplinar e interdisciplinar desde los ámbitos de la salud, la educación y los servicios sociales. La coordinación entre los diferentes servicios es fundamental para la detección y atención precoz de las dificultades en el desarrollo de los niños.

El programa de seguimiento de niños grandes prematuros, llevado a cabo de manera conjunta en Móstoles (Madrid) por el hospital, el Centro de Atención
Temprana AFANDEM (Asociación de Familias y Amigos de las Personas con Discapacidad Intelectual de Móstoles) y los equipos de orientación locales, se enmarca dentro de esta perspectiva.

Este programa conjunto nace de la preocupación de los profesionales de los diferentes servicios tras escuchar una ponencia sobre niños prematuros en las Jornadas de Alto Riesgo organizadas por el Real Patronato para la Discapacidad en 1996. En el curso escolar 1996-1997, el Equipo de Atención Temprana de AFANDEM y el Hospital de Móstoles iniciaron la coordinación, a la que se incorporó durante el curso 2001-2002 el Equipo de Orientación Educativa de Móstoles. A partir de 2005 se sumaron los pediatras de los centros de salud de la localidad.

Los objetivos de este programa son:

- Detectar precozmente las necesidades educativas de los niños de este grupo de población, así como de su modalidad educativa.

- Contribuir a la optimización de los recursos y los medios destinados a la prevención y detección precoz de niños con deficiencias o en situaciones de riesgo, así como a su atención lo más temprana posible.

- Coordinación entre los diferentes servicios que atienden a dichos niños y a sus familias. 
Población y coordinación entre niveles

Se hace el seguimiento de los niños con antecedentes personales de gran prematuridad (al nacer: peso $\leqslant 1.500 \mathrm{~g}$ y/o duración del embarazo $\leqslant 32$ semanas).

Los niños seguidos por los servicios sanitarios, los de estimulación y los educativos no coinciden en su totalidad, ya que hay niños que nacen en el Hospital de Móstoles y que cambian posteriormente de residencia, así como niños que nacen en otros hospitales y que se escolarizan o reciben tratamiento en esta localidad. En la actualidad compartimos el $50 \%$ de los casos.

Las derivaciones y el intercambio de información se hacen de forma fluida entre el hospital, los centros de salud, el Centro de Atención Temprana AFANDEM y los equipos de orientación (figura 1), y se pueden describir así:
- Los equipos de orientación -Equipo de Atención Temprana (EAT) y Equipo de Orientación Educativa y Psicopedagógica- hacen el seguimiento de las acciones educativas que necesitan los niños y las áreas escolares donde presentan dificultades.

- El centro de atención temprana lleva a cabo los programas de estimulación.

- Los servicios sanitarios realizan el seguimiento desde el ámbito de la salud.

Se mantiene, al menos, una reunión de coordinación anual para intercambiar los datos de seguimiento y las informaciones recogidas. Para la derivación se usan los siguientes protocolos habituales: parte de interconsulta del hospital, formulario modelo PADI e informe de evaluación. Asimismo, disponemos de

Figura 1. Interrelación entre el hospital, los pediatras de Atención Primaria, el Centro de Atención Temprana AFANDEM y los equipos de orientación.

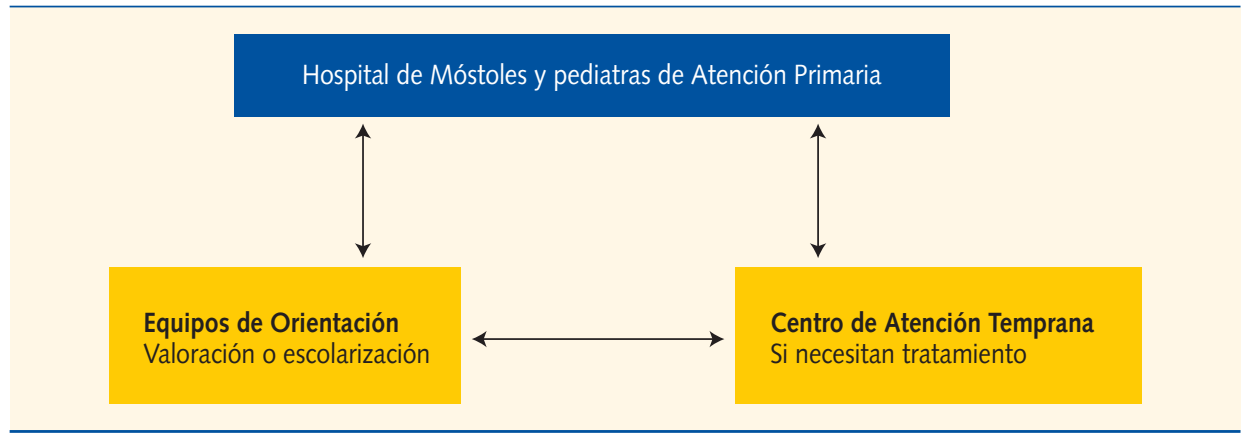


una base de datos conjunta para poder analizar los datos.

\section{Seguimiento desde el ámbito sanitario}

El nacimiento prematuro es aquel que tiene lugar antes de las 37 semanas completas de edad gestacional (o 259 días). En la mayoría de los países se está apreciando un significativo aumento en los últimos años'. En EE. UU., entre los años 1981-1994, el porcentaje de nacimientos prematuros pasó del 9 al 10,5\%, con bolsas poblacionales que presentan casi un $15 \%$ de prematuridad $^{3}$. En la figura 2 se muestra el porcentaje de prematuridad en la Comunidad de Madrid ${ }^{4}$ (años 19862005), en el que se observa un aumento progresivo a partir de 1996. La figura 3 muestra el porcentaje de prematuridad en el Hospital de Móstoles (Madrid) en los años 1999-2006, que oscila entre un 6,1 y un $8 \%$ sobre un promedio de 1.800 partos anuales. Los nacimientos prematuros representan el $35-40 \%$ de los ingresos en la Unidad de Neonatología de este hospital.

Los diferentes grados de prematuridad pueden clasificarse desde el punto de vista de la edad gestacional: pretérmino tardío (33-36 semanas), medio (25-32 semanas) y extremo (menos de 25 semanas); o bien tomando como referencia el peso en el momento del nacimiento: prematuro de muy bajo peso (1.000-1500 g; VLBW según la terminología anglosajona) y prematuro de peso extremadamente bajo (menos de $1.000 \mathrm{~g}$; ELBW), entendiendo que todo recién nacido de peso inferior a $2.500 \mathrm{~g}$ se clasifica como de bajo peso al nacer (LBW).

Las estrategias utilizadas en el tratamiento perinatal del niño prematuro (esteroides prenatales, antibióticos, monitorización, surfactante, nacimiento por cesárea, asistencia respiratoria especial, ingreso en unidades de cuidados intensivos, etc.) han propiciado un aumento muy notable de la supervivencia de los niños de muy bajo peso. Datos del National Institute of Child Health and Human Development (EE. UU.) destacan una supervivencia del $85 \%$ para los niños de entre 501 y $1.500 \mathrm{~g}$ nacidos en 1997-2002 Los trabajos que comparan diferentes períodos de tiempo ${ }^{6}$ constatan este hecho, si bien, a la hora de valorar las secuelas halladas en el desarrollo, en algunas publicaciones se señala que la disminución de la mortalidad en los grupos de peso más bajo podría relacionarse con un aumento notable de alteraciones del desarrollo y la discapacidad7. En EE. UU., la prematuridad está presente en el $45 \%$ de los niños con parálisis cerebral, en el $35 \%$ con alteraciones visuales y en el $25 \%$ con alteraciones auditivas y cognitivas ${ }^{8}$. 
Figura 2. Evolución de la prematuridad en la Comunidad de Madrid (años 1986-2005) (porcentaje sobre el total de nacimientos).

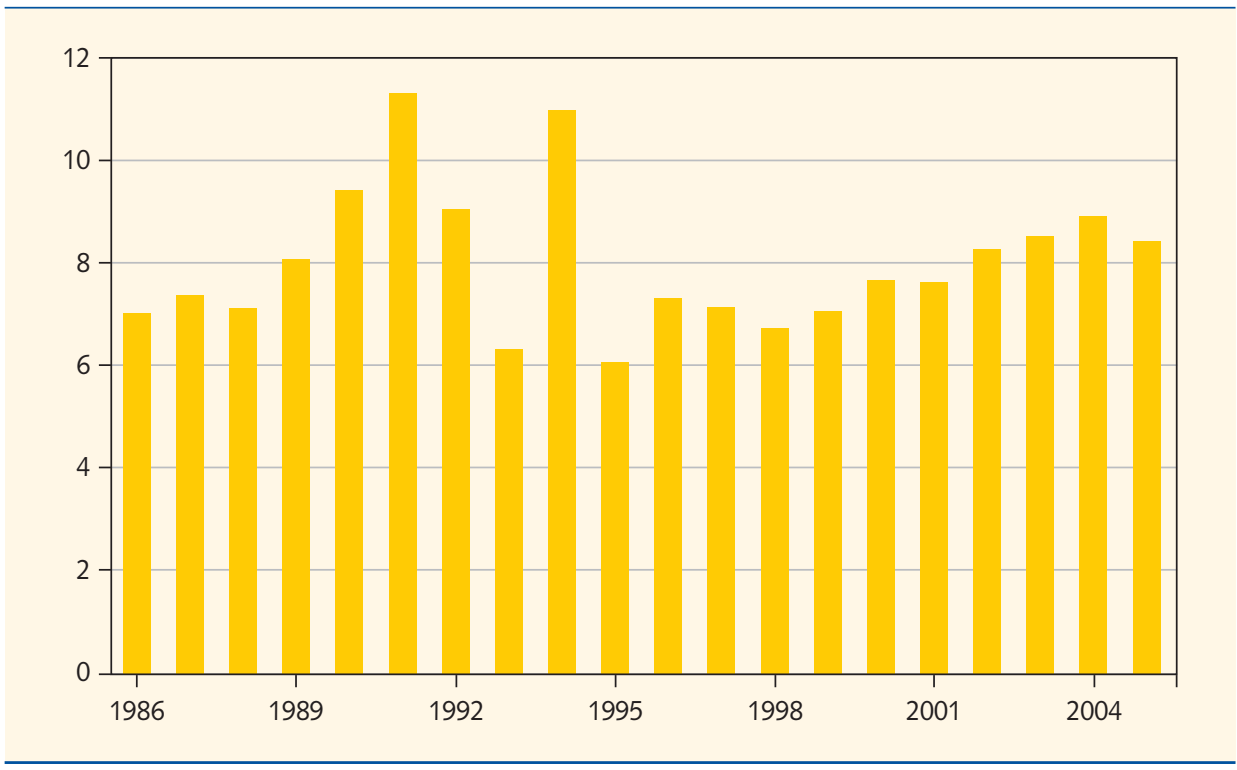

Figura 3. Evolución de la tasa de prematuridad en la maternidad del Hospital de Móstoles (años 1999-2006) (porcentaje sobre el total de nacimientos).

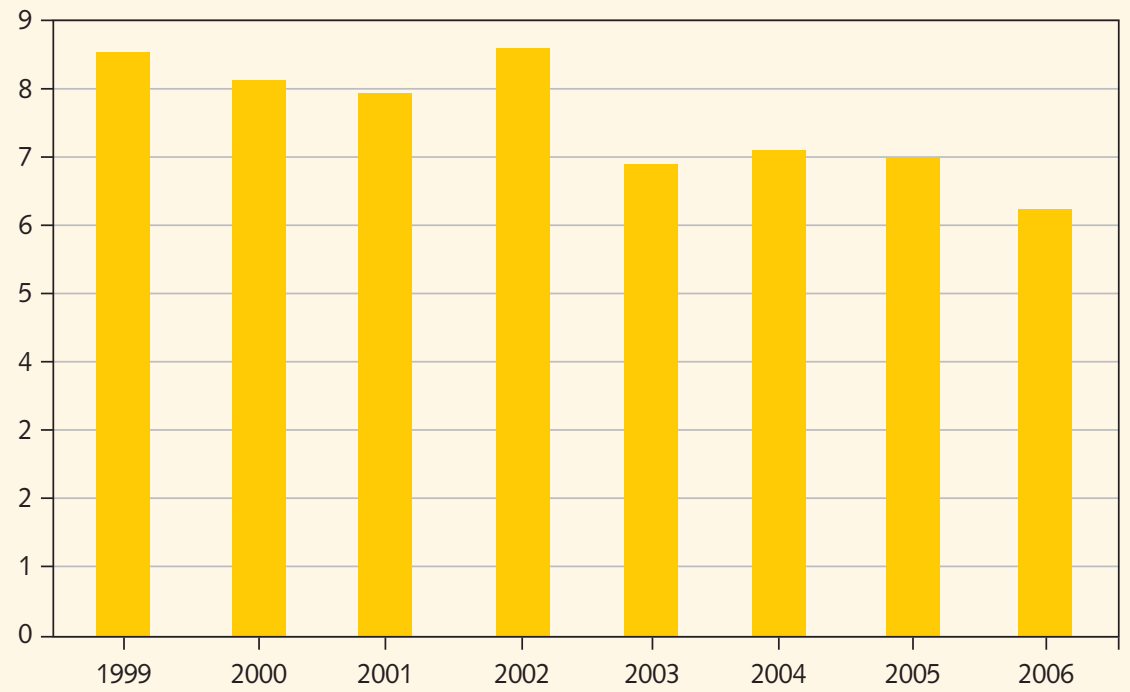


Los acontecimientos de tipo médico, como la hemorragia intracraneal, la retinopatía, la enteritis necrotizante, la infección nosocomial, la leucomalacia periventricular y algunas complicaciones relacionadas con los tratamientos, como la displasia broncopulmonar, influyen de forma decisiva en el desarrollo neurocognitivo, $0^{90}$. En más del $85 \%$ de los ni- ños nacidos prematuramente que tienen parálisis cerebral, ésta se debe a una hemorragia en el sistema nervioso central. La parálisis cerebral en sus distintas formas, no todas graves, es $15-20$ veces más frecuente en los prematuros de peso inferior a $1.500 \mathrm{~g}(30-120 / 1.000)$ que en la población general pediátrica (2-2,5/1.000 en países desarrollados);

Tabla 1. Grados de discapacidad en los niños nacidos prematuramente

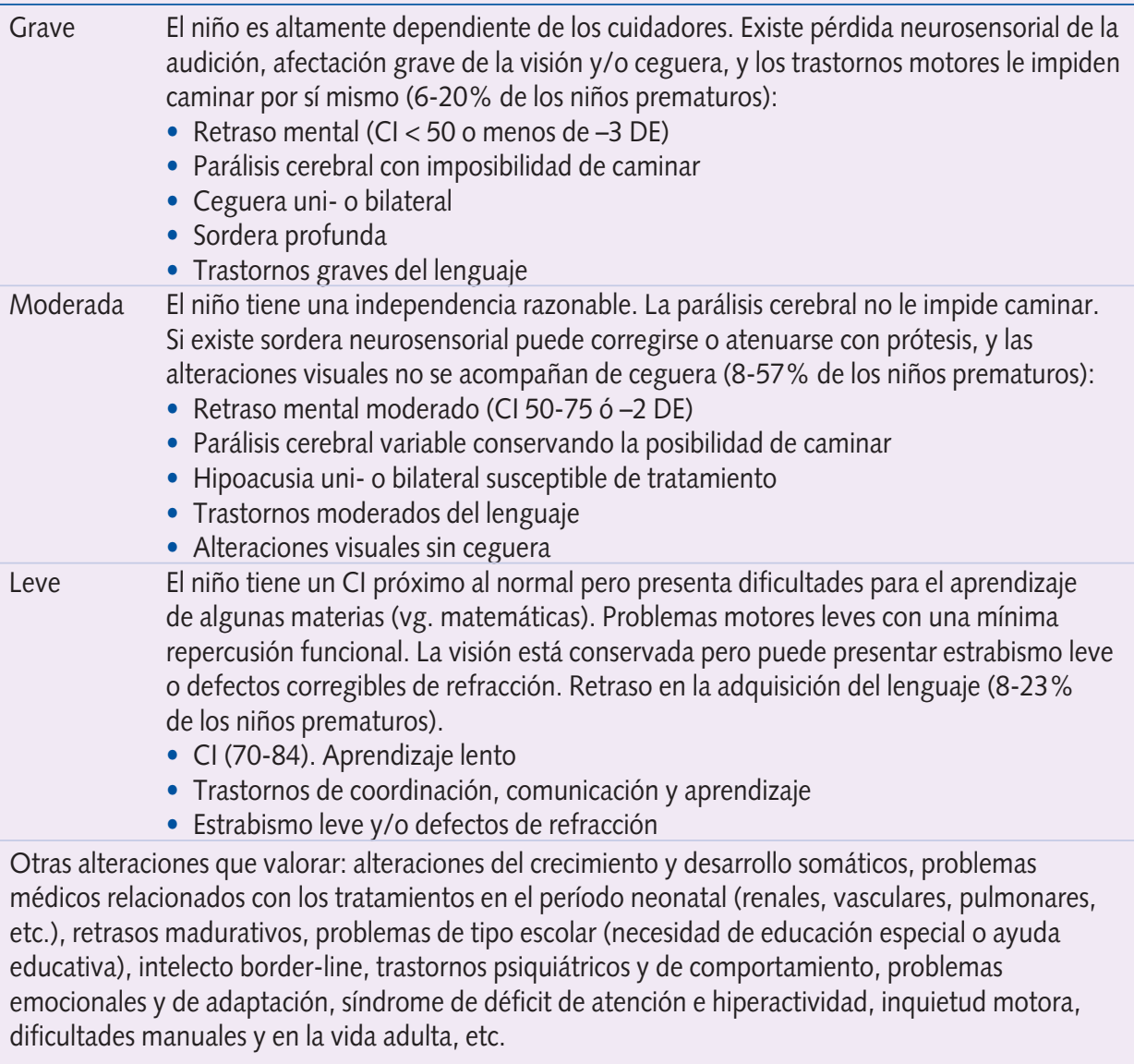

Cl: coeficiente (cociente) intelectual; DE: desviación estándar. 
incluso el déficit en el crecimiento en la fase de ingreso hospitalario actúa como un factor adverso de relación independiente ${ }^{11}$. Además, estas poblaciones de niños nacidos antes del término de la gestación tienen con mayor frecuencia enfermedades crónicas, un mayor número de ingresos hospitalarios en los primeros años de vida, asma, problemas nutricionales, reflujo gastroesofágico y mayor incidencia de muerte súbita.

Dentro del programa conjunto, el Servicio de Pediatría del Hospital de Móstoles tiene establecido un programa de seguimiento de niños de peso inferior a
1.500 g o edad gestacional inferior a 32 semanas. En la tabla 1 se expone la clasificación de los diferentes grados de discapacidad empleada, que coincide en general con lo admitido en la bibliografía (se suele reservar el término "minusvalía" para los trastornos que limitan la realización de actividades cotidianas típicas de los niños de su edad y el de "deficiencia" para las limitaciones en las actividades sociales).

Debemos señalar que, a la hora de comparar los diferentes trabajos publicados, hay que tener en consideración, entre otros aspectos: la época en la que se

Tabla 2. Seguimiento de 213 recién nacidos prematuros (peso inferior a 1.500 g) nacidos en el período 1989-2005 y valorados en el año 2007

\begin{tabular}{|c|c|c|c|}
\hline Secuelas & $\mathrm{N}$ & Peso (g) & Edad gestacional (semanas) \\
\hline Ninguna secuela & $154 / 185(83,2 \%)$ & $1.171+/-227$ & $29,6+/-5,6(p<0,01)$ \\
\hline Con secuelas & $31 / 185(16,7 \%)$ & $1.039+/-243$ & $20,1+/-6,9(p<0,01)$ \\
\hline $\begin{array}{l}\text { Valoración cuantitativa } \\
\text { de las secuelas }\end{array}$ & $\begin{array}{l}\text { Parálisis cerebral: } 1 \\
\text { Retraso mental sol } \\
\text { Parálisis cerebral + } \\
\text { Alteraciones visual } \\
\text { - Estrabismo: 31/ } \\
\text { - Miopía/astigma } \\
\text { - Ceguera monola } \\
\text { - Microftalmía: 1/ } \\
\text { - Desprendimient } \\
\text { Alteraciones auditi } \\
\text { - Hipoacusia mon } \\
\text { - Retraso en el len } \\
\text { - Tartamudez: 2/ } \\
\text { Otras alteraciones } \\
\text { litiasis biliar, escolic }\end{array}$ & $\begin{array}{l}\text { /185 (7,5\%) } \\
\text { : } 11 / 185(5,9 \%) \\
\text { etraso mental: } 4 \\
\text { s: } \\
85(16,7 \%) \\
\text { smo: } 32 / 185(17 \\
\text { eral: } 2 / 185(1 \% \\
85(0,5 \%) \\
\text { de retina: } 3 / 185 \\
\text { as: } \\
\text { lateral: } 2 / 185(1 \\
\text { uaje: } 10 / 185 \text { (5 } \\
85 \text { (1\%) } \\
\text { ncontradas: sínd } \\
\text { sis grave, trastorr }\end{array}$ & $\begin{array}{l}185(2,1 \%) \\
2 \%) \\
(1,6 \%) \\
4 \%) \\
\text { rome de West, riñón único, } \\
10 \text { psiquiátrico esquizoide. }\end{array}$ \\
\hline
\end{tabular}


extrae la muestra poblacional (las estrategias de tratamiento de la prematuridad han variado con el paso de los años), las características de las poblaciones, la selección según el peso o la edad gestacional, las herramientas utilizadas para la valoración y el tiempo de seguimiento.

En la tabla 2 mostramos la morbilidad encontrada en 213 prematuros nacidos en el período 1989-2005 incluidos en nuestro programa de seguimiento, valorados en el año $2007^{12}$ y seguidos hasta una edad promedio de 7,9+/-4,2 años con un porcentaje de pérdidas del $13 \%$ $(n=185)$. Aparte de otras consideraciones, se observa cómo las alteraciones visuales son las más frecuentes; la parálisis cerebral representa el 7,5\% de la serie, y el retraso mental, el $5,9 \%$. Con respecto al peso, en los menores de $1.000 \mathrm{~g}$ se halló un $10,2 \%$ de parálisis cerebral frente a un $6,4 \%$ en los niños de peso entre 1.000-1.500 g. En la revisión cita$\mathrm{da}^{12}$ observamos cómo los niños de me- nor peso y edad gestacional tuvieron una mayor incidencia de secuelas (con secuelas: peso: $1.039+/-243 \mathrm{~g}$; edad gestacional: $26,06+/-6,9$ semanas; $\sin$ secuelas: peso: $1.171,2+/-247 \mathrm{~g}$; edad gestacional: $29,7+/-5,6$ semanas; $p<$ 0,01 para el peso y la edad gestacional). En el estudio de las variables relacionadas con la evolución se mostraban el desprendimiento placentario, la ecografía cerebral patológica, la necesidad de asistencia respiratoria y el peso inferior a 1.000 g como las más relacionadas con el mal pronóstico (tabla 3). En conjunto, como se señala en la tabla 2 , desde el punto de vista médico, casi un $85 \%$ de los niños seguidos tuvo una evolución normal hasta la edad del fin del seguimiento y un $17 \%$ aproximadamente presentó algún tipo de daño físico o alteración en el desarrollo.

La experiencia en el seguimiento de los niños prematuros permite establecer en los programas las pautas tanto de

\begin{tabular}{|c|c|c|c|}
\hline Variables & $p$ & $S$ & $E$ \\
\hline 1. Desprendimiento de placenta & $p<0,1$ & Baja & Alta \\
\hline 2. Ecografia cerebral patológica & $p<0,001$ & Baja & Alta \\
\hline 3. Asistencia respiratoria & $p<0,01$ & Alta & Baja \\
\hline 4. Peso $<1.000 \mathrm{~g}$ versus $>1.000 \mathrm{~g}$ & $p=0,05$ & Baja & Baja \\
\hline 5. Reanimación tipos IV/V & $p<0,05 / p<0,01$ & Alta & Baja \\
\hline 6. Administración de oxígeno al $100 \%$ & $p<0,01$ & Baja & Media \\
\hline S: sensibilidad; E: especificidad. & & & \\
\hline
\end{tabular}


Tabla 4. Características y cronología de las discapacidades

\begin{tabular}{ll}
\hline Alteraciones motoras & - El $90 \%$ se detecta en los 2 primeros años; los casos no graves, más tarde \\
- Los casos leves pueden resolverse \\
Alteraciones cognitivas & Pueden ser independientes de las motoras \\
- Se clasifican mejor a partir de los 2 años \\
Alteraciones visuales \\
- Debidas a daño neurológico o retinopatía \\
- El desprendimiento de retina es predictivo de ceguera \\
- La retinopatía moderada/media puede acompañarse de miopía, estrabis- \\
mo, astigmatismo y anisometropía
\end{tabular}

diagnóstico como de actuación, contando siempre con un equipo multidisciplinar que valore todos los matices del desarrollo (tabla 4).

Pero en la actualidad hay dos aspectos en los que se centran la mayoría de los trabajos publicados. El primero de ellos es el rendimiento y la adaptación en la época escolar; el segundo es el estado de estos niños en la vida adulta. Los comentaremos de forma muy breve.

Con respecto al primero, se están estudiando, cada vez con más precisión, los aspectos relativos a la escolarización en las épocas de la infancia y la adolescencia. Incluso corrigiendo factores como el estado socioeconómico y marital, la educación materna y el sexo, se han hallado en estos niños puntuaciones más bajas en lectoescritura y test matemáti$\cos ^{13}$. La evaluación del neurodesarrollo en la época escolar es mejor apreciada por el hecho de que el psicólogo cuenta con instrumentos de diagnóstico más seguros y veraces que en épocas anteriores de la edad infantil. Por otra parte, como elemento favorable, existen algunas evidencias de que, con la edad, el desarrollo cerebral del niño podría mejorar e incluso, en algunos supuestos, recuperar el déficit cognitivo ${ }^{14}$. Si bien el bajo rendimiento escolar está relacionado con numerosos factores, en las poblaciones de niños prematuros, el primero de estos factores es el grado de prematuridad. Los niños de peso inferior a $1.500 \mathrm{~g}$ y de 
edad gestacional inferior a 32 semanas no solamente tienen una mayor incidencia de secuelas físicas, sino que también presentan con una mayor frecuencia evaluaciones escolares pobres, mayores requerimientos de ayuda educativa, problemas de tipo psicológico, depresión, ansiedad e hiperactividad y déficit de atención y concentración ${ }^{9,10}$.

Aunque los estudios de seguimiento se centran en los niños de peso y edad gestacional menores, se ha comprobado que los prematuros de 32-36 semanas o prematuros cerca del término de la gestación (late preterm) tienen también un riesgo nada despreciable de presentar déficits neurocognitivos $y$, por lo tanto, de rendimiento escolar deficiente cuando se comparan con los niños que nacieron al término de la gestación².

La prematuridad podría tener un potencial impacto sobre la vida adulta ${ }^{15,16}$. Si bien no en todos los trabajos se señala este hecho ${ }^{17}$, en muchos de ellos se admite que los adultos que fueron prematuros tendrían, por una parte, una mayor incidencia de problemas médicos (resistencia a la insulina, hipertensión arterial, disminución de la capacidad reproductora, mayor riesgo de tener hijos prematuros en las mujeres ${ }^{18 ;}, y$, por otra, el nacimiento prematuro podría asociarse con niveles educativos bajos, con la imposibilidad de vivir independientemente, así como con dificultades para conservar el empleo ${ }^{19}$. También se ha señalado que las relaciones con los padres serían más difíciles y que, en el caso de las mujeres, la ansiedad, la depresión y los problemas de atención son más frecuentes al compararlas con la población general $15,16,17,18,19,20,21,22$.

Pero hemos de señalar que no todos los autores opinan de la misma forma. Saigal et al. ${ }^{20}$, aún admitiendo un mayor número de dificultades entre los adultos que nacieron prematuros, no encuentran diferencias a los 23 años de edad en cuanto al nivel académico, la educación secundaria, el empleo, la vida independiente y el matrimonio o aspectos relativos a la paternidad. Estos autores valoran la calidad de vida de estos adultos y sus familias $y$, a pesar de las limitaciones y la complejidad en sus actividades, el parámetro calidad de vida no es diferente de la población control tanto en el adulto como en sus familias ${ }^{21}$.

\section{Seguimiento desde el ámbito terapéutico. Centro de Atención Temprana AFANDEM}

Las derivaciones a nuestro centro pueden ser realizadas por el hospital de referencia, los pediatras de Atención Primaria, el centro base, los equipos educativos y la propia familia. 
Una vez recibido el caso se realiza una valoración inicial:

- Se valoran las necesidades de la familia a través de la recogida de información mediante una entrevista familiar por parte del trabajador social.

- Se realiza una entrevista a la familia de carácter semiabierto por parte del psicólogo en la que se recoge la información necesaria para entender el entorno familiar que rodea al niño, así como las conductas que presenta y la forma de interacción familiar.

- Se valoran las necesidades del niño desde el punto de vista psicológico a través de pruebas estandarizadas, la escala de desarrollo de Brunet-Lezine hasta los 30 meses y el cuestionario de madurez neuropsicológica infantil con niños de 3-6 años. Utilizamos esta prueba porque nos permite valorar el desarrollo madurativo y mide los signos neurológicos menores, especialmente localizados en la corteza cerebral, ya que el incremento en número e intensidad de éstos es significativo de disfunción cerebral. Consta de ocho escalas: psicomotricidad, lenguaje, atención, estructuración espacial, visopercepción, memoria, estructuración rítmico-temporal y lateralidad.

- Valoración de las necesidades del niño por parte del fisioterapeuta.

Existen varias líneas de intervención posterior: programas de seguimiento, tratamiento y de apoyo familiar.

\section{Programa de seguimiento}

Se incluirán en este programa los siguientes casos:

- Los menores de 12 meses cuando la diferencia entre la edad de desarrollo y la cronológica corregida no sea superior a dos meses.

- Los mayores de 12 meses cuando la diferencia entre la edad de desarrollo y la cronológica no sea superior a dos meses, no teniendo en cuenta la edad corregida por prematuridad.

Como norma, los seguimientos se realizan cuatrimestralmente; sin embargo, a veces existen excepciones debido a que los niños llegan muy tempranamente, encontrándose en un mes con edad corregida y no quedando claro ni para el psicólogo ni para el fisioterapeuta la situación de ese bebé, o bien porque la familia está tan desbordada por la situación que requiere un seguimiento más a corto plazo. 


\section{Programa de tratamiento}

En este programa se incluirán los casos cuyo desfase entre la edad cronológica y la edad de desarrollo sea superior a dos meses.

Nuestro centro, como cualquier otro centro de atención temprana, está constituido por un equipo multidisciplinar formado por especialistas en el ámbito psicológico, rehabilitador y social. Está integrado por trabajadores sociales, psicólogos, logopedas, estimuladores (los profesionales en nuestro centro tienen doble perfil, son especialistas en logopedia y estimulación, con la finalidad de reducir referentes para el niño), psicoterapia, fisioterapia y psicomotricidad. La intervención se planifica en equipo en las reuniones de coordinación interna que se mantienen con una frecuencia semanal, de manera que se programa de forma individual, teniendo en cuenta las necesidades y posibilidades de cada niño en cada área de desarrollo, así como a su familia y su entorno.

En un primer momento, en la mayoría de los casos reciben fisioterapia y estimulación global y del lenguaje, una o dos veces por semana, dependiendo de la edad y las necesidades del niño y su familia. En los casos en que el niño tiene una edad inferior a seis meses, la estimulación es llevada a cabo por el Departa- mento de Psicología, con la finalidad de poder trabajar a la vez el vínculo entre madre e hijo. Posteriormente, si fuese necesario, podrán recibir otros tratamientos como logopedia, psicomotricidad y psicoterapia.

En general, estos niños de alto riesgo, cuando reciben el alta en el Departamento de Fisioterapia, pasan al de Psicomotricidad con la finalidad de mejorar la inmadurez de los grupos musculares, lo que debería facilitarles su control postural y la coordinación de los músculos de la locomoción. El sistema postural está constituido por las informaciones de las posturas y del equilibrio, y se construye y desarrolla basándose en las informaciones visoespacial y vestibular, de manera que el desarrollo del sistema postural posibilita la integración de los aprendizajes en prematuros.

Las ventajas de trabajar con un equipo multidisciplinar son fundamentales a la hora de sacar adelante a los niños con estas características, ya que permite trabajar todas las áreas del desarrollo desde los diferentes departamentos con un abordaje común: resolver los problemas de coordinación ojo-mano, paliar los trastornos del esquema corporal, los trastornos posturales, los trastornos de la percepción que implicarán disfunciones en los sistemas de la organización 
espacio-temporal y los trastornos del lenguaje, que dificultan la comunicación y entorpecen los procesos sociales.

No podemos olvidar que todas estas características mencionadas van acompañadas por una serie de dificultades visuales que habrá que trabajar a modo de prevención, para evitar, en la medida de lo posible, una confusión con la lateralidad, futuras inquietudes motoras que pueden dar lugar a falsos diagnósticos de hiperactividad o, en determinados casos, ocasionar una falta de visión binocular, con la consecuente repercusión en sus futuros aprendizajes escolares, teniendo en cuenta que cualesquiera de estos trastornos son suficientes, por sí solos, para plantear dificultades en el aprendizaje escolar posterior del niño prematuro.

Destacar que, desde un centro de atención temprana, la intervención que se realiza va más dirigida a estimular la base de los aprendizajes, de manera que se lleva a cabo un trabajo diferente, pero paralelo, al efectuado por los apoyos escolares.

\section{Programa de apoyo familiar}

La acogida a la familia que llega con un gran prematuro es una de las fases más importantes. Para las familias que reciben el impacto de un problema semejante supone una crisis, que en un principio es desorganizadora de su equi- librio. Sin embargo, no debemos entender su evolución como predeterminada, pudiendo, como toda situación crítica, resolverse con el beneficio de una mayor madurez y un enriquecimiento del núcleo familiar.

Debemos tener en cuenta que las primeras 72 horas de vida son muy importantes para los padres: son momentos de tristeza, impotencia y desesperación. Los primeros días son muy duros; tienen preguntas sin respuestas, miedo, sentimientos de culpa. Pasan las semanas y la angustia por la supervivencia poco a poco da paso a la obsesión por las secuelas. Aparecen entonces nuevas preguntas: ¿cómo será el futuro desarrollo del niño?, ¿estará condicionado por sus limitaciones intelectuales, físicas, somáticas o sensoriales?

Podemos encontrarnos con padres siempre apenados que tienden a delegar parte de sus funciones relativas al cuidado a diversos profesionales, que los orientan y aconsejan. La actitud de los técnicos no debe ser directiva, para evitar que su capacidad cuidadora quede mermada o anulada.

En otras ocasiones, los padres adoptan una actitud de mucha protección, en la que lo son todo para el niño, de manera que esto genera tensiones y bloquea la adquisición de una adecuada autonomía. Autonomía no es lo mismo que abando- 
no. El equilibrio está en educar para que el niño, aún dependiente, necesite cada vez menos a la madre, sin que esto signifique perder relación o cuidados, sino cambiar la forma de recibirlos. Los profesionales precisan unas cualidades que van más allá de la cualificación profesional; necesitan sensibilidad, empatía y seguridad en sí mismos para establecer las bases de una relación que se apoya, además, en una actitud de disponibilidad.

Los profesionales trabajamos con hipótesis. El diagnóstico y el pronóstico están siendo cuestionados día a día y, sobre todo, cada niño, sea cual sea su problema, y cada familia poseen una individualidad con la que no funcionan las recetas de cocina.

La familia espera del profesional, al menos, un diagnóstico y una información lo más veraces posible sobre las posibilidades del niño. Hay que tener en cuenta que esta información va a repercutir de maneras distintas según el momento en que se dé, la personalidad de la familia y el apoyo recibido, no sólo en el momento del contacto inicial, sino también a largo plazo.

La escucha es una cuestión técnica muy importante, tanto para el diagnóstico como para el tratamiento. Hay que destacar, tras el diagnóstico, los aspectos positivos y las posibilidades, más que las limitaciones. Todo esto permite a los padres verbalizar fantasías y temores que pueden ser elaborados. No podemos olvidar que una información objetiva se complementa con un apoyo adecuado. Los padres necesitan tiempo para elaborar el golpe psicológico, de manera que permanezca en ellos la esperanza, pero sin alentar falsas expectativas. Toda esta serie de acontecimientos provoca tensiones en la pareja, que habrá que contrarrestar con el apoyo mutuo y el diálogo. Sin embargo, no siempre es suficiente el apoyo desde el Departamento de Psicología; es entonces cuando son derivados a terapia de familia, que puede ser ofrecida en el mismo centro.

También hay que tener en cuenta que existen otros componentes de la familia que se ven implicados:

- Los hermanos que reaccionan con sentimientos de responsabilidad, temor por su salud y hostilidad hacia sus padres, que parecen alejarse y olvidarlos a veces, mientras que aumentan las exigencias respecto a ellos. Sin embargo, pueden aportar una colaboración muy útil para el desarrollo de su hermano a la vez que se fortalecen sus sentimientos de utilidad. En nuestro centro se realizan talleres de her- 
manos todos los años como vía de elaboración de sus dificultades.

- Los abuelos también pueden aportar una gran ayuda aliviando las tensiones y haciendo una función de relevo en muchas ocasiones. Esta participación por su parte les sirve también a ellos para sentirse útiles y aliviar sus propias tensiones. El centro dispone también del servicio Taller de Abuelos.

La supervivencia de estos niños de muy bajo peso al nacer se encuentra estrechamente asociada con un alto riesgo de presentar problemas de salud y desarroIlo. Es un factor de riesgo para el desarrollo cognitivo y neuropsicológico durante la infancia, ya que su desarrollo neuromadurativo, sobre todo en las áreas de asociación de la corteza cerebral, resulta afectado. Hasta el momento se ha confirmado el incremento de deficiencia mental, retraso psicomotor, trastornos del lenguaje, hiperactividad, trastornos de lateralidad y problemas de conducta. Estudios realizados con anterioridad con niños de muy bajo peso indican una clara predisposición a presentar dificultades específicas de aprendizaje al inicio de la etapa escolar. Los trastornos de lateralidad y visopercepción son muy frecuentes, según los estudios efectuados en nuestro país por Mulas y Retortillo.
Para terminar, hay que destacar que cualquier intervención no tendría sentido si no existiese una coordinación externa entre todos los profesionales que trabajan con el niño, como vía de posibilitar un mayor rendimiento en sus resultados.

\section{Seguimiento desde el ámbito} educativo

Los EAT y los equipos educativos tienen, entre sus funciones, la detección de necesidades educativas especiales, así como la orientación y el apoyo a los alumnos que las poseen. Atendemos a estos alumnos según sean sus necesidades con diferentes respuestas educativas. Éstas se ajustan tras realizar una evaluación psicopedagógica de dichos alumnos.

\section{Modalidades}

Las distintas modalidades de respuesta en el ámbito educativo son:

- Ordinaria: alumnos que siguen su escolaridad sin ningún tipo de dificultades o secuelas.

- Dificultades de aprendizaje: alumnos que requieren seguimiento por sus dificultades de aprendizaje $y / 0$ que acuden a refuerzo escolar. Suelen cursar con retraso en los aprendizajes escolares de forma global o en algún área (lengua, matemáti- 
cas, etc.). Tienen dificultades asociadas a retraso mental límite, déficit de atención y retraso motor. Equivalen a alumnos con minusvalía leve desde el área médica.

- Alumnos con necesidades educativas especiales: alumnos que están en centros ordinarios con uno de los siguientes diagnósticos. Equivalen a niños con minusvalía moderada desde el área médica:

- Discapacidad psíquica: retraso mental, trastornos generalizados del desarrollo.

- Trastornos del desarrollo del lenguaje: de la expresión y/o de la comprensión.

- Trastornos del comportamiento y de las emociones de comienzo habitual en la infancia y en la adolescencia: hiperactividad con déficit de atención, trastorno disocial.

- Discapacidad sensorial: pérdida o desviación significativa de la vista y funciones relacionadas, pérdida o desviación significativa de las funciones auditivas y vestibulares.

- Discapacidad motora: pérdida o desviación significativa de las funciones neuromusculoesqueléticas y relacionadas con el movimiento.
- Otras circunstancias: plurideficiencias, retraso madurativo (en la etapa de educación infantil).

- Alumnos en centros de educación especial: alumnos con grandes discapacidades que requieren centros muy especializados. Equivalen a niños con minusvalía grave desde el área médica.

\section{Evaluación y seguimiento}

Con los alumnos con dificultades de aprendizaje o necesidades educativas especiales se hace un seguimiento para conocer en qué áreas son fuertes y en cuáles débiles.

Tanto para la evaluación psicopedagógica como para el seguimiento se utilizan pruebas estandarizadas (que miden capacidades) y diagnósticos médicos, para tratar de evitar otras variables que afectan al conjunto de la población (curriculares, grupos familiares, grupo aula, grupo amigos, etc.), aunque estos factores se deberán tener presentes como complemento.

El EAT hace la evaluación y el seguimiento si el alumno no está escolarizado en un colegio o está en una escuela infantil, procurando llevarlos a cabo a los $8,14,20$ y 30 meses de edad. El Equipo de Orientación realizará el seguimiento una vez escolarizado en el colegio. 
Se utilizan las siguientes pruebas: Brunet Lezine hasta los 30 meses, MSCA en la escuela infantil hasta los cinco años y WISC-R en $2 .^{\circ}$ y $6 .^{\circ}$ de Primaria. Se pueden complementar con: competencia curricular proporcionada por el tutor al finalizar ciclos de Primaria; el seguimiento familiar, usando cuestionarios o entrevistas; y el seguimiento afectivo y social, empleando el TAMAI en $6^{\circ}$ de Primaria o con cuestionarios a tutores.

Los equipos de orientación tienen, al menos, dos reuniones: una al principio de curso para actualizar el listado de alumnos en seguimiento y otra al final para recoger y analizar los datos obtenidos durante el curso.

A modo de resumen:

- La población de prematuros es pequeña respecto al conjunto de niños.

- Es significativo el número de prematuros que necesitan ayuda escolar (el 44\% aproximadamente).

- La detección precoz y la intervención temprana favorecen el desarrollo global de estos niños.

- Con la ayuda necesaria (médica, educativa, social, familiar), la mayoría de estos niños serán personas autónomas (un 3,7\% precisan educación especial).

\section{Conclusiones y propuestas de futuro}

Todo lo señalado nos conduce a resaltar que las poblaciones de niños prematuros tienen, a lo largo de su vida, mayores necesidades, no solamente desde el punto de vista sanitario sino también educacional, social y psicológico, necesidades que se amplían a su familia (la intervención sobre los padres puede favorecer la evolución neurocognitiva). Es, por lo tanto, imprescindible establecer programas de seguimiento coordinados que incluyan ayuda al crecimiento, ayuda escolar, servicios de la comunidad y conexión con el cuidado primario. En definitiva, ayudas de un gran número de profesionales que permitan el diagnóstico precoz de los retrasos del desarrollo y de las discapacidades, y el control y tratamiento adecuados.

En los próximos años los objetivos serán: avanzar en la coordinación entre los diferentes servicios para intentar que el seguimiento alcance a la totalidad de estos niños, mejorar la base de datos para poder realizar análisis más cualitativos, profundizar en las áreas donde presentan dificultades en el ámbito escolar, incluir factores de riesgo (tabaco, alcoholismo, hipoestimulación, etc.) y crear grupos de apoyo a las familias de niños prematuros. 


\section{Bibliografía}

1. Plan de Acción para Personas con Discapacidad de la Comunidad de Madrid, 2005-2008. Madrid: Consejería de Familia y Asuntos Sociales; 2005 [consultado el 12/03/2010]. Disponible en www.madrid.org

2. Chyi LJ, Lee HC, Hintz SR. School outcomes of late preterm infants: special needs and challenges for infants born at 32 to 36 weeks gestation. J Pediatr. 2008;153:25-31.

3. Goldenberg RL, Rouse DJ. Prevention of Premature Birth. N Engl J Med. 1998;339:313-20.

4. Morbilidad hospitalaria. Madrid, 2007. Bol Epidemiol Comunidad de Madrid. 2008;14(10): 3-53.

5. Fanaroff AA, Stoll BJ, Wright LL. Trends in neonatal morbidity and mortality for very low birthweight infants. Am J Obstet Gynecol. 2007; 196:e1-8.

6. Vohr BR, Wright LL, Poole WK, Mc Donald SA. Neurodevelopmental outcomes of extremely low birth weight infants $<32$ weeks' gestation between 1993 and 1998. Pediatrics. 2005;116: 635-43.

7. Hintz SR, Kendrick DE, Vohr BR. Changes in neurodevelopmental outcomes at 18 to 22 months' corrected age among infants of less than 25 weeks' gestational age born in 1993-1999. Pediatrics. 2005;115:1645-51.

8. Allen MC. Neurodevelopmental outcomes of preterm infants. Curr Opin Neurol. 2008;21: 123-8.

9. Eichenwald EC, Stark AR. Management and outcomes of very low birth weight. N Engl J Med. 2008;358:1700-11.

10. Neubauer AP, Voss W, Kattner E. Outcome of extremely low birth weight survivors at school age: the influence of perinatal parameters on neurodevelopment. Eur J Pediatr. 2008;167:87-95.
11. Ehrenkranz RA, Dusick AM, Vohr BR. Growth in the neonatal intensive care unit influences neurodevelopmental and growth outcomes of extremely low birth weight infants. Pediatrics. 2006;117:1253-61.

12. García Martín A, Herencia Anillo C, Rico Vales T, Puyol Buil P, Bote Llorente CJ. Intercambio de experiencias EAT-EOEP. La orientación: investigación y acción. La prematuridad como causa de discapacidad en el niño. Madrid: Universidad Rey Juan Carlos; 2007.

13. Breslau N, Paneth NS, Lucia VC. The lingering academic deficits of low birth weight children. Pediatrics. 2004;114:1035-40.

14. Ment LR, Vohr B, Allan W. Change in cognitive function over time in very low-birth-weight infants. JAMA. 2003;289:705-11.

15. Hack $M$, Klein N. Young adult attainments of preterm infants. JAMA. 2006;295:695-6.

16. Hille ET, Weisglas-Kuperus N, Van Goudoever JB. Functional outcomes and participation in young adulthood for very preterm and very low birth weight infants: the Dutch Project on Preterm and Small for Gestational Age Infants at 19 years of age. Pediatrics. 2007;120: e587-95.

17. Dalziel SR, Lim VK, Lambert A. Psychological functioning and health-related quality of life in adulthood after preterm birth. Dev Med Child Neurol. 2007;49:597-602.

18. Hovi P, Andersson S, Eriksson JG. Glucose regulation in young adults with very low birth weight. N Engl J Med. 2007;356:2053-63.

19. Swamy GK, Ostbye T, Skjaerven R. Association of preterm birth with long-term survival, reproduction and next-generation preterm birth. JAMA. 2008;299:1429-36.

20. Saigal S, Stoskopf B, Streiner D, Boyle M, Pinelli J, Paneth $\mathrm{N}$, et al. Transition of extremely lowbirth-weight infants from adolescence to young 
adulthood: comparison with normal birth-weight controls. JAMA. 2006;295:667-75.

21. Saigal S, Stoskopf B, Pinelli J. Self-perceived health-related quality of life of former extremely low birth weight infants at young adulthood. Pediatrics. 2006;118:1140-8.
22. Treyvaud K, Anderson VA, Howard K, Bear M, Hunt RW, Doyle LW, et al. Parenting behaviour is associated with the early neurobehavioral development of very preterm Children. Pediatrics. 2009;123:555-61. 